the primary tumour. Finally, Receiver operating characteristics (ROC) curves were generated to confirm predicting the value of plasma-based miR148a in HCC.

Results Plasma-based miR-148a expression was significantly lower in 155 HCCs compared to 96 liver cirrhosis $(p<0.01)$ as well as 95 healthy control $(p<0.01)$. Upon removal of the primary HCC tumour, levels of plasma-based miR-48aincreased significantly compared with their initial levels $(\mathrm{p}<0.0001)$. The area under receiver operating characteristic (AUROC) curve for plasma-based miR-148a was 0.919, with a sensitivity of $89.6 \%$ and specificity of $89.0 \%$ for HCC patients compared with liver cirrhosis. In HCC with negative or less expressing AFP, the AUROC values of plasma-based miR148a were 0.949 with a sensitivity of $90.6 \%$ and specificity of $92.6 \%$.

Conclusions Plasma-based miR-148a can be applied as a potential non-invasive biomarker for HCC screening, especially for HCC with negative or less expressing AFP, which can make up AFP deficiency in predicting HCC occurrence.

Notes This project was funded by Application Project of Clinical Features in Capital City (Z141107002514507).

\section{IDDF2018-ABS-0133 PERFORMANCE OF NON-INVASIVE BLOOD PARAMETERS FOR DETECTING SIGNIFICANT LIVER FIBROSIS IN PATIENTS WITH CHRONIC HEPATITIS B}

Shivam Khare* , Anil Arora, Praveen Sharma, Shashi Dhawan. Sir Ganga Ram Hospital, New Delhi, India

\subsection{6/gutjnl-2018-IDDFabstracts.217}

Background Since liver biopsy is invasive and Fibroscan has limited availability, various non-invasive blood parameters need evaluation to find the most useful parameter for detection of significant fibrosis in patients with chronic hepatitis $\mathrm{B}(\mathrm{CHB})$.

Methods In this retrospective study, records of patients who underwent liver biopsy for treatment naive $\mathrm{CHB}$, were evaluated to obtain various non-invasive blood parameters (ASTplatelet ratio index[APRI], Fibrosis-4[Fib-4], GGT-platelet ratio [GPR], GGT-to-albumin ratio[GAR]), in addition to Fibroscan, to detect significant fibrosis and compared these with fibrosis stage of liver biopsy.

\begin{tabular}{|c|c|c|c|c|c|c|c|c|}
\hline Parameter & Formula & $\mathrm{N}$ & AUROC & $95 \% \mathrm{Cl}$ & $\begin{array}{c}P \\
\text { value }\end{array}$ & $\begin{array}{c}\text { Best } \\
\text { cut-off }\end{array}$ & PPV & NPV \\
\hline APRI & $\begin{array}{l}\text { (AST/ULN) } \\
{ }^{*} 100 / \mathrm{Plt}\end{array}$ & 107 & 0.687 & $\begin{array}{c}0.533- \\
0.840\end{array}$ & 0.030 & 0.935 & $33 \%$ & $93 \%$ \\
\hline Fib-4 & $\begin{array}{c}\left(\mathrm{Age}^{*} \mathrm{AST}\right) / \\
\left(\mathrm{Plt}{ }^{\star} ? \mathrm{ALT}\right)\end{array}$ & 107 & 0.735 & $\begin{array}{c}0.590- \\
0.880\end{array}$ & 0.006 & 2.324 & $37 \%$ & $93 \%$ \\
\hline GPR & $\begin{array}{l}\text { (GGT/ULN) } \\
{ }^{*} 100 / \mathrm{Plt}\end{array}$ & 91 & 0.727 & $\begin{array}{c}0.577- \\
0.876\end{array}$ & 0.015 & 0.444 & $28 \%$ & $95 \%$ \\
\hline GAR & GGT/Alb & 90 & 0.688 & $\begin{array}{c}0.516- \\
0.860\end{array}$ & 0.037 & 17.848 & $35 \%$ & $92 \%$ \\
\hline Fibroscan & - & 92 & 0.768 & $\begin{array}{c}0.633- \\
0.902\end{array}$ & 0.009 & 5.35 & $17 \%$ & $100 \%$ \\
\hline
\end{tabular}

Results A total of 125 were included (median age 34 [range 11-82] years, 74\%males). Most (83\%) patients were $\mathrm{HBeAg}$ negative. Liver biopsy revealed nil/mild fibrosis (Ishak $<3$ ) in $87 \%$ patients and significant fibrosis (Ishak $\geq 3$ ) in $13 \%$ patients. Among non-invasive blood parameters, APRI and Fib4 were available in 107 patients, GPR in 91, and GAR in 90. Fibroscan was available in 92 patients. All the blood parameters, as well as Fibroscan, were able to detect significant fibrosis significantly well $(\mathrm{p}<0.05)$. All parameters had PPV $<35 \%$ but NPV above 92\%. Fibroscan had the highest NPV $(100 \%)$ at a cut-off $<5.35 \mathrm{kPa}$, and among the blood parameters, GPR had highest NPV $(95 \%)$ at a cut-off $<0.444$ (table 1).

Conclusions Non-invasive blood parameters (APRI, Fib-4, GPR, and GAR) with NPVs above 92\% are excellent parameters for ruling-out significant fibrosis in patients with $\mathrm{CHB}$. Among the blood parameter, GPR has the best NPV of $95 \%$ at a cut-off below 0.444 and should be used when Fibroscan is not available.

\section{IDDF2018-ABS-0137 LONGITUDINAL ASSESSMENT OF ALPHA- FETOPROTEIN, LECTIN-REACTIVE ALPHA- FETOPROTEIN, AND DES-GAMMA-CARBOXY PROTHROMBIN FOR THE EARLY DETECTION OF HEPATOCELLULAR CARCINOMA}

Jonggi Choi*, Sang Young Lee, Young-Suk Lim, Woochang Lee, Sail Chun. Asan Medical Center, University of Ulsan College of Medicine, Korea, South

\subsection{6/gutjnl-2018-IDDFabstracts.218}

Background Few studies for biomarkers in hepatocellular carcinoma (HCC) have reported using prospectively collected longitudinal samples.

Methods Among 689 patients who participated to four randomised trials for cirrhosis and/or chronic hepatitis B, 42 HCC cases were diagnosed during follow up, and 168 controls were matched for age, sex, aetiology, cirrhosis, and duration of follow-up in a 1:4 ratio. Samples at the time of HCC diagnosis, months -6 , and month -12 were tested for alpha-fetoprotein (AFP), lectin-reactive AFP (AFP-L3), and des-gammacarboxy prothrombin (DCP) in the HCC cases and controls.

Results Of 42 cases with HCC, 39 (93\%) had cirrhosis, 36 $(85.7 \%)$ had normal alanine aminotransferase, and 31 (73.8\%) had single $\mathrm{HCC}<2 \mathrm{~cm}$ (38 [90.5\%] within Milan criteria). AFP and AFP-L3 started to increase from 6 months before diagnosis of HCC $(p<0.05)$, while remained unchanged in the controls. The area under the receiver operator characteristic curves (AUROCs) for AFP, AFP-L3, and DCP at month 0 were $0.77,0.73$, and 0.71 , respectively. Combining AFP and AFP-L3 showed higher AUROC of 0.83 at HCC diagnosis, while including DCP did not further improve performance (AUROC, 0.86). With the optimal cutoff values (AFP, $5 \mathrm{ng} / \mathrm{mL}$ and AFP-L3, 4\%), sensitivity and specificity of combined AFP and AFP-L3 were $79 \%$ and $87 \%$, respectively.

Conclusions AFP and AFP-L3 increased from 6 months before the diagnosis of early-stage HCC. AFP and AFP-L3 combination showed better performance than single biomarkers. Adopting a cutoff value of AFP level at $5 \mathrm{ng} / \mathrm{mL}$ would significantly increase the sensitivity for HCC detection. 


\section{IDDF2018-ABS-0138 COMPARISON OF THE LONG-TERM OUTCOMES OF ENDOSCOPIC PAPILLARY LARGE-BALLOON DILATION ALONE VERSUS ENDOSCOPIC SPHINCTEROTOMY FOR REMOVAL OF BILE DUCT STONES}

Wenying Yang*. Ruijin Hospital, Affiliated to Shanghai Jiao Tong University School of Medicine, China

\subsection{6/gutjnl-2018-IDDFabstracts.219}

Background Endoscopic papillary large-balloon dilation (EPLBD) is an alternative to endoscopic sphincterotomy (EST) for treatment of common bile duct (CBD) stones. However, limited data exist regarding the comparison of the long-term outcomes for these techniques. In this study, we aimed to compare the long-term outcomes after EST with those after EPLBD alone for removal of CBD stones.

Methods The records of patients with EST or EPLBD alone referred for CBD stones retrieval between June 2008, and July 2015 were retrospectively reviewed. Complete stone clearance, ERCP-related adverse events, and late biliary complications during long-term follow-up were analysed.

Results Basic patient characteristics were similar between the groups that underwent EST $(n=60)$ and EPLBD alone $(n=161)$. EPLBD alone compared with EST resulted in similar outcomes in terms of complete stone clearance $(99.4 \%$ vs $100 \%, \mathrm{p}=0.54)$ and ERCP-related adverse events $(6.8 \%$ vs $10.0 \%, \mathrm{p}=0.41)$. The mean duration of the follow-up was 74.5 months and 71.6 months who underwent EST and EPLBD alone, respectively $(p=0.42)$. Late biliary complications were frequently occurred in the EST group than EPLBD alone group (11 [18.6\%] vs 16 [10.2\%]), although the difference did not reach statistical significance $(p=0.11)$. Furthermore, the incidence of cholangitis without stone recurrence was significantly higher in the EST group than EPLBD alone group (5.1\% vs $0 \%, \mathrm{p}=0.02)$.

Conclusions As an alternative to EST, EPLBD has similar efficacy and safety for managing CBD stones. During longterm follow-up, patients who underwent EPLBD alone may have less late biliary complications compared with those after EST.

\section{IDDF2018-ABS-0144 SINGLE-OPERATOR PERORAL CHOLANGIOSCOPY IN THE TREATMENT OF DIFFICULT BILIARY STONES: A SYSTEMATIC REVIEW AND META-ANALYSIS}

${ }^{1}$ Zheng Jin*, ${ }^{2}$ Xiaofeng Zhang. 'Hangzhou Geriatric Hospital, Hangzhou First People's Hospital Group, Hangzhou, China; ${ }^{2}$ Department of Gastroenterology, Hangzhou First People's Hospital, Nanjing Medical University, Hangzhou, China

\subsection{6/gutjnl-2018-IDDFabstracts.220}

Background Current evidence supporting the utility of singleoperator peroral cholangioscope (SOPOC) in the management of difficult bile duct stones is limited. We conducted the present systemic review and meta-analysis to evaluate the efficacy and safety of SOPOC in the treatment of difficult bile duct stones.

Methods A search of studies up to January 2018 was acquired, using MEDLINE, EMBASE, the Cochrane Library and Google Scholar. Quality assessment of the studies was completed with a modified Newcastle-Ottawa Scale. The main outcomes of interest were single-session stone clearance rate, complete stone clearance rate and adverse events. We calculated the pooled proportions with random-effects models. Subgroup analyses were also performed based on SOPOC type.

Results A total of 21 studies involving 2490 patients met the inclusion criteria. Average number of stones per patient was 2.2. Mean stone size was $17.4 \mathrm{~mm}$. The pooled proportion of patients with single-session stone clearance was $72.5 \% \quad(95 \%$ confidence interval [95\% CI], 63.2\%-80.9\%). (Figure 1). Complete stone clearance was achieved in 94.7\% (95\% CI, 90.1\%-98.1\%) of patients with median endoscopic sessions of 2.0. The pooled adverse event rate was 5.5\% (95\% CI, $3.3 \%-8.2 \%)$. For SOPOC type SpyGlass and SpyGlass DS, single-session stone clearance rate was 69.1\% (95\%CI: $56.3 \%$ $80.6 \%)$ and $80.0 \%$ (95\% CI: $75.8 \%-83.9 \%)$, respectively. Complete stone clearance rate was $92.8 \%$ (95\%CI: $86.5 \%$ 97.5\%) and 96.7\% (95\%CI: 88.1\%-100.0\%), respectively. Adverse events rate was 6.2\% (95\%CI: $3.4 \%-9.7 \%)$ and $3.0 \%$ (95\% CI: $1.8 \%-4.4 \%)$, respectively.

Conclusions SOPOC is an effective and safe management option for the treatment of bile duct stones when conventional methods have failed. More randomised controlled trials 